\title{
ORIGINAL
}

\section{CONSUMO DE HIPOLIPEMIANTES EN ESPAÑA, 1987-2000}

\author{
Miguel Siles Gutiérrez (1), Margarita Goldaracena Tanco (1), Luisa Ávila Muñoz (2) y Belén \\ Crespo Sánchez-Eznarriaga (2) \\ (1) Inspección Farmacéutica. Dirección Territorial del Insalud. Zaragoza. \\ (2) Área de Gestión de Farmacia. Subdirección General Atención Primaria. Insalud.
}

\section{RESUMEN}

Fundamento: Los medicamentos hipolipemiantes se encuentran en la actualidad entre los más usados y de mayor repercusión sobre el gasto farmacéutico del Sistema Nacional de la Salud. Al mismo tiempo, dentro del grupo se producen constantes cambios de unas moléculas por otras nuevas.

Métodos: Se estudiaron todas las prescripciones realizadas en receta del Sistema Nacional de Salud del subgrupo terapéutico B04A «Preparados Hipolipemiantes» en el conjunto de España durante el período 1987 a 2000. Los diferentes principios activos se han agrupado de acuerdo a los subgrupos de la Anatomical Therapeutic Chemical Classification. Los parámetros de medida usados son el número de envases, el importe económico, el número de Dosis Diarias Definidas y el Coste Tratamiento Día.

Resultados: El consumo total de hipolipemiantes ascendió durante este período a 389.133 millones de pesetas (ptas) (2.339 millones de euros) y 2.580 millones de Dosis Diarias Definidas. El consumo de cada año superó al del anterior, oscilando desde 4.504 millones de ptas en 1987 a 71.389 millones de ptas en el 2000; y desde 63,6 a 420,9 millones de Dosis Diarias Definidas. El Coste Tratamiento Día se incrementó a su vez desde 71 a 170 ptas. El subgrupo de mayor consumo fue el de las estatinas con el $73,4 \%$ del consumo total en importe y el $54,8 \%$ en número de Dosis Diarias Definidas, y con tendencia a aumentar

Conclusiones: Los medicamentos hipolipemiantes han experimentado un incremento notable y constante durante el período analizado, no sólo en importe económico, sino también en número de Dosis Diarias Definidas. La aparición de las estatinas ha modificado las pautas de uso del grupo.

Palabras clave: Colesterol. Hipolipemiantes. Estudios de Utilización de Medicamentos. DDD. DHD. ATC.

Correspondencia:

Miguel Siles Gutiérrez

Dirección Territorial Insalud Zaragoza

Paseo María Agustín, 16

50004 Zaragoza

Correo electrónico: msiles@dtz.insalud.es

\section{ABSTRACT}

\section{Lipid-Reducing Drug Consumption in Spain, 1987-2000}

Background: Lipid-reducing drugs are currently among those most used and which have the greatest impact on the drug spending of the Spanish National Health Care System. At the same time, constant changeovers are taking place from one type of molecule to another within this group.

Methods: A study was made of all of the Spanish National Health Care System prescriptions filled from the treatment subgroup B04A «Lipid-Reducing Preparations» nationwide throughout the 1987-2000 period. The different active ingredients have been grouped according to the subgroups of the Anatomical Therapeutic Chemical Classification. The measurement parameters employed were the number of packages, the economic cost, the number of Defined Daily Doses (DDD) and the Treatment/Day Cost (TDC).

Results: Lipid-reducing drug consumption for the timeframe in question totals $389,133,314,142$ pesetas $(2,338,738,320$ euros) and 2,580,179,790 DDD. Consumption rose annually, ranging from $4,504,321,869$ pesetas in 1987 to $71,389,377,528$ in 2000; and from $63,594,576$ to $420,878,797$ DDD. The TDC rose, in turn from 71 to 170 pesetas. The subgroup showing the highest consumption was that of the statin drugs, totalling $73.4 \%$ of the total consumption by cost and $54.8 \%$ of the number of DDD's, showing a trend toward further growth.

Conclusions: Lipid-reducing drugs have undergone a noticeable, constant rise throughout the timeframe under analysis, not only regarding the price, but also the number of DDD's. Statin drugs having started to be used has modified the guidelines of use for this group.

Keywords: Cholesterol. Lipid-reducing drug. Drug Use Studies. DDD's. DHD. TDC. 


\section{INTRODUCCIÓN}

Las enfermedades cardiovasculares son la primera causa de muerte en nuestro país, al igual que ocurre en el resto de países industrializados, con tasas entorno al $40 \%$ del total de fallecimientos ${ }^{1-8}$. Desde hace tiempo se sabe que el colesterol plasmático es uno de los principales factores de riesgo, por lo que su control se ha convertido en una medida preventiva de primer orden, para la cual, además de la elección de una dieta adecuada y ciertos hábitos de vida saludables, como no fumar y practicar ejercicio físico ${ }^{9,10}$, se cuenta con un grupo específico de medicamentos: los hipolipemiantes o antiateromatosos.

Los primeros hipolipemiantes aparecieron en los años sesenta, y pertenecían al grupo de los fibratos. Posteriormente se fueron incorporando otros grupos de moléculas, como los secuestrantes de ácidos biliares, derivados del ácido nicotínico, heparinoides, etc. Pero fue a comienzos de los años noventa cuando se produjo la auténtica revolución en la terapia antihipercolesterolemiante con la aparición en el mercado de la primera estatina, o inhibidor de la hidroximetil-glutaril-coenzima A - $\mathrm{HMGCoA-}$ reductasa. Desde entonces hasta hoy se ha ido incrementando el número de tratamientos con este subgrupo de hipolipemiantes, tanto por el aumento del número de pacientes tratados, como por haber sustituido en gran medida a aquellos fármacos más antiguos. Y puesto que una de las características de las estatinas es su elevado precio, su irrupción en la terapéutica de esta enfermedad ha supuesto un importante impacto en el coste farmacéutico global.

Estos fármacos reducen los niveles de colesterol de lipoproteínas de baja densidad (Col-LDL) y pueden prevenir la formación de lesiones ateroescleróticas, lentificar su proceso y producir su regresión, mejorar la vasodilatación coronaria y reducir la mortalidad por cardiopatía coronaria. El tratamiento con todos estos fármacos general- mente se mantiene indefinidamente, ya que de suspenderse la colesterolemia vuelve a los niveles previos al tratamiento ${ }^{11}$.

La elección del hipolipemiante concreto no ha estado exenta de polémica, puesto que mucho antes de que se demostrara la efectividad de la primera estatina, la simvastatina $^{12}$ en 1994, ya se usaba como de primera elección por la mayoría de los médicos. La situación actual es que sólo dos moléculas han demostrado su efectividad: además de la simvastatina ya citada, la pravastatina ${ }^{13,14}$. Al mismo tiempo, la relativa efectividad queda a menudo en entredicho ante el elevado coste que conlleva su utilización ${ }^{1}$. Por otra parte, el manejo de la enfermedad ha de comenzarse siempre por medidas de tipo dietético, el abandono del tabaquismo y la realización de ejercicio físico, previamente al tratamiento farmacológico ${ }^{15,16}$. Las recomendaciones terapéuticas actuales son la elección de las estatinas como hipolipemiantes de primera línea, y los fibratos para casos de hipertrigliceridemia o bajo nivel de HDL, tanto para el Programa Nacional Norteamericano sobre Educación Sanitaria y Colesterol, como para la Sociedad Europea y Española de Ateroesclerosis ${ }^{15-18}$.

Resulta evidente que en las sociedades occidentales, con un alto índice de envejecimiento de la población, sedentarización y otros hábitos de riesgo para las enfermedades cardiovasculares, la utilización de medicamentos hipolipemiantes tiene gran importancia, tanto por la repercusión sobre la salud como por la asignación de recursos que se le debe destinar.

Es por esto que creemos que el estudio de la utilización de este grupo de fármacos durante un largo período de tiempo en el conjunto del país es de interés, por cuanto permite conocer la proporción de cada una de las diferentes moléculas dentro del subgrupo terapéutico, su evolución y el coste económico que representan dentro del consumo farmacéutico global. Asimismo, con el adecuado parámetro de medida de ese consu- 
mo, se pueden establecer comparaciones con otros países, así como tener una idea aproximada del número de pacientes en tratamiento. En nuestro estudio hemos analizado todas las prescripciones realizadas en España durante los catorce años que van desde 1987 a 2000.

\section{MATERIAL Y MÉTODOS}

Se analizaron todas las dispensaciones realizadas en receta médica oficial del Sistema Nacional de la Salud durante los años 1987 a 2000. Los datos de consumo farmacéutico se obtienen mediante el proceso de facturación de las recetas médicas efectuado en cada provincia en el que se toma, entre otros datos, el código nacional de la especialidad farmacéutica; estos datos se centralizan en el Ministerio de Sanidad y Consumo en la base de datos ESPES, de la que hemos obtenido el consumo agregado de cada especialidad a nivel nacional. En nuestro caso, hemos tratado estos datos con un programa informático específico creado por la Inspección Farmacéutica del Insalud de Zaragoza, el cual permite asignar el principio activo a cada especialidad, y a partir de aquí, el análisis del consumo agregado por principios activos.

Para el estudio de los principios activos se ha adoptado la clasificación internacional de la Organización Mundial de la Salud, Anatomical Therapeutic Chemical Classification (ATC), y se ha usado como parámetro de medida, además del número $\left(n .^{\circ}\right)$ de envases y el importe a precio de venta al público (PVP), el número de Dosis Diarias Definidas (DDD) consumidas. La DDD se define como la unidad técnica de medida y comparación que equivale a la dosis media diaria de mantenimiento cuando se utiliza en su indicación principal, por una vía de administración determinada, expresada en cantidad de principio activo ${ }^{19}$. Los valores de referencia usados son los publicados por el WHO Collaborating Centre for Drug Statistics Methodology (Anatomical Therapeu- tic Chemical Classification Index, 2.000) ${ }^{19}$. Los valores no establecidos por este organismo se han fijado de acuerdo a las pautas recomendadas en la bibliografía actual ${ }^{20-23}$, y se recogen en la tabla 1.

Para cada presentación comercial consumida se ha calculado primero la cantidad de principio activo en unidades físicas (generalmente en $\mathrm{mg}$ ) que contiene un envase; dividiendo esta cantidad por la DDD de ese principio activo en esa vía de administración, expresada en esa misma unidad, se obtiene el n. ${ }^{\circ}$ de DDD que contiene cada envase; y multiplicando esa cifra por el n..$^{\circ}$ de envases consumidos de esa especialidad, se tiene el $n .{ }^{\circ}$ de DDD de esa especialidad. Al sumar todas las DDD de todas las especialidades que contienen un mismo principio activo, se obtiene el $n .^{\circ}$ de DDD de un principio activo:

$\mathrm{CPA}=$ Cantidad de principio activo de una especialidad $=$ dosis $\quad$ unidades de contenido

$\mathrm{CPADDD}=\mathrm{n} .^{\circ}$ DDD de una especialidad $=$ $=\mathrm{CPA} / \mathrm{DDD}$ del principio activo por esa vía

\section{N. ${ }^{\circ}$ DDD de la especialidad $=$ CPADDD} n. ${ }^{\circ}$ envases de la especialidad
N. ${ }^{\circ}$ DDD principio activo $=\quad$ N. ${ }^{\circ}$ DDD de todas las especialidades con ese principio activo

Para poder hacer comparaciones de consumo entre períodos de tiempo o ámbitos poblacionales diferentes, hemos usado el n. ${ }^{\circ}$ de DDD / 1.000 Habitantes / Día (DHD) ${ }^{24-27}$.

$$
\begin{gathered}
\text { n. }{ }^{\circ} \mathrm{DHD}=\left(\mathrm{n}^{\mathrm{o}} \mathrm{DDD} 1000\right) / \\
(365 \text { población }) .
\end{gathered}
$$

El Coste Tratamiento Día (CTD) es el importe a PVP en pesetas (ptas) de una DDD, y se ha usado para comparar el coste de las di- 
Tabla 1

Valores de la DDD(1) de Principios Activos no contemplados en la ATC

\begin{tabular}{|c|c|c|c|}
\hline Principio activo & $D D D(1)$ & Unidad (2) & Autor (3) \\
\hline Clofibrato Aluminio & $2.000,00$ & $\mathrm{mg}$ & FT \\
\hline Etofibrato & 900,00 & $\mathrm{mg}$ & MD \\
\hline Clofibrida & $1.350,00$ & $\mathrm{mg}$ & FT \\
\hline Clofibrato en asociación & 4,00 & UDO & FT \\
\hline Binifibrato & $1.800,00$ & $\mathrm{mg}$ & MD \\
\hline Plafibrida & $1.600,00$ & $\mathrm{mg}$ & MD \\
\hline Pirifibrato & $1.500,00$ & $\mathrm{mg}$ & MD \\
\hline Clofibrato + Piricarbato & 2,00 & UDO & FT \\
\hline Sitofibrato & $2.000,00$ & $\mathrm{mg}$ & FT \\
\hline Tocofibrato & 800,00 & $\mathrm{mg}$ & MD \\
\hline Clofibrato Aluminio en asociación & 2,00 & UDO & FT \\
\hline Dextrano Dietilaminoetil & $2.500,00$ & $\mathrm{mg}$ & MD \\
\hline Filicol & $6.000,00$ & $\mathrm{mg}$ & FT \\
\hline Nicotinico Ácido + Pentosanopolisulfúrico & 1,00 & UDP & FT \\
\hline Nicotinato de Tocoferol & 450,00 & $\mathrm{mg}$ & FT \\
\hline Nicofibrato & $1.000,00$ & $\mathrm{mg}$ & FT \\
\hline Nicoclonato & $1.000,00$ & $\mathrm{mg}$ & FT \\
\hline Piricarbato & $1.000,00$ & $\mathrm{mg}$ & FT \\
\hline Pirozadilo & $1.500,00$ & $\mathrm{mg}$ & FT \\
\hline Nicotinato Tocoferol en Asociación & 4,00 & UDO & FT \\
\hline Piricarbato en asociación & 5,00 & UDO & FT \\
\hline Nicotinato Xantinol + Mucopolisacárido & 3,00 & $\mathrm{mg}$ & FT \\
\hline Probucol & $1.000,00$ & $\mathrm{mg}$ & MD \\
\hline Tiadenol & $1.200,00$ & $\mathrm{mg}$ & MD \\
\hline Benfluorex & 450,00 & $\mathrm{mg}$ & MD \\
\hline Dextrotirosina & 4,00 & $\mathrm{mg}$ & FT \\
\hline Sulodexida & 36,00 & $\mathrm{mg}$ & FT \\
\hline Pantetina & 600,00 & $\mathrm{mg}$ & FT \\
\hline Heparinoide & 100,00 & $\mathrm{mg}$ & FT \\
\hline Sultosilato de Piperazina & $1.500,00$ & $\mathrm{mg}$ & FT \\
\hline Condroitinsulfúrico Ácido & $3.000,00$ & $\mathrm{mg}$ & FT \\
\hline Amilasa + Lipasa + Tirosinasa & 1,00 & UDP & FT \\
\hline Fosfolipidos & 30,00 & $\mathrm{mg}$ & FT \\
\hline Fosfolipidos en asociación & 6,00 & UDO & FT \\
\hline
\end{tabular}

(1) DDD: Dosis Diaria Definida.

(2) UDO: Unidad de Dosificación Oral (comprimidos, cápsulas, etc.). UDP: Unidad de Dosificación Parenteral (ampollas, viales, etc.)

(3) MD: Martindale 32ed. FT: Ficha Técnica del producto.

ferentes alternativas terapéuticas dentro del subgrupo.

Aunque, como se ha indicado, se trata de datos de dispensación o, mejor aún, de facturación de recetas por las oficinas de farmacia al Sistema Nacional de la Salud, en nuestro estudio hablaremos indistintamente de dispensaciones, de prescripciones o, simplemente, de consumo. Por la misma razón, no se considera el consumo producido dentro de los hospitales, ni el consumo mediante receta privada o de regímenes especiales de la Seguridad Social, como MUFACE, ISFAS o MUGEJU, ni el consumo que se haya podido producir sin receta médica. To- 
das las cifras de valoración económica están expresadas en importe a PVP en pesetas.

La población considerada es la publicada por el Instituto Nacional de Estadística. En realidad, la población que hubiéramos debido considerar es la que recibe la asistencia a través del Sistema Nacional de Salud; pero estos datos no están disponibles y consideramos que es aceptable asimilarla al total de la población española, por cuanto la casi totalidad de la misma está cubierta por el Sistema.

\section{RESULTADOS}

El consumo de hipolipemiantes en España durante el conjunto del período de estudio, los catorce años que van desde 1987 a 2000, fue de 389.133 millones de pesetas (2.339 millones de euros) y 2.580 millones de DDD, correspondientes a 108 millones de envases. El consumo se incrementó cada año respecto del anterior en todos los parámetros de medida, multiplicándose a lo lar- go del período por 15,85 el consumo en importe y por 6,62 en n. ${ }^{\circ}$ de DDD. Las cantidades halladas para cada año, incluidas las de $n .^{\circ}$ de envases consumidos, se recogen en la tabla 2. En ella también puede verse que la evolución del CTD fue claramente ascendente, si bien con algunos matices de interés que se comentan en el apartado de Discusión, entre los que destaca el punto de inflexión hacia arriba de principios de los años 90, justo cuando aparecieron las estatinas, $y$ la estabilización al final del período de estudio. De principio a fin, el CTD se multiplicó por 2,40; sólo en el año 2000 se produjo una disminución respecto del año anterior.

A fin de conocer la evolución durante el período de estudio de los diferentes tipos de hipolipemiantes, se calculó el consumo anual de cada subgrupo de la clasificación ATC, obteniéndose los resultados que aparecen en la figura 1. Se aprecia el arranque de las estatinas a partir de su aparición en el mercado en 1990, así como un importante punto de inflexión a partir de 1998, que se comenta más adelante. La evolución de los fibratos es un

Tabla 2

Consumo de Hipolipemiantes en España, 1987-2000, evolución por años

\begin{tabular}{|rrrrrrrr|}
\hline Año & $N .^{\circ}$ envases & N. ${ }^{\circ}$ DDDs & $\begin{array}{c}N .^{o} \\
\text { DHDs }\end{array}$ & Importe ptas. & Importe euros & CTD (2) \\
\hline 1987 & 3.370 .510 & 63.594 .576 & 4,5 & 4.504 .321 .869 & 27.071 .520 & 71 \\
1988 & 3.855 .037 & 81.588 .267 & 5,8 & 6.338 .732 .464 & 38.096 .549 & 78 \\
1989 & 4.120 .840 & 96.440 .447 & 6,7 & 7.673 .929 .040 & 46.121 .242 & 80 \\
1990 & 4.640 .480 & 111.704 .547 & 7,7 & 9.613 .814 .180 & 57.780 .187 & 86 \\
1991 & 5.315 .130 & 123.005 .500 & 8,4 & 14.350 .255 .508 & 86.246 .773 & 117 \\
1992 & 5.999 .856 & 138.436 .252 & 9,8 & 19.451 .132 .561 & 116.903 .661 & 141 \\
1993 & 6.538 .709 & 150.935 .486 & 10,6 & 22.432 .931 .915 & 134.824 .636 & 149 \\
1994 & 7.254 .815 & 166.977 .420 & 11,5 & 25.245 .401 .914 & 151.727 .921 & 151 \\
1995 & 7.892 .672 & 182.633 .951 & 12,4 & 29.116 .028 .311 & 174.990 .854 & 159 \\
1996 & 8.458 .926 & 197.717 .830 & 13,4 & 32.546 .582 .276 & 195.608 .899 & 165 \\
1997 & 9.316 .171 & 217.842 .839 & 15,0 & 35.941 .468 .957 & 216.012 .579 & 165 \\
1998 & 11.361 .819 & 276.636 .159 & 19,1 & 48.301 .489 .780 & 290.297 .800 & 175 \\
1999 & 13.888 .728 & 351.787 .719 & 24,2 & 62.227 .847 .839 & 373.996 .898 & 177 \\
2000 & 16.036 .835 & 420.878 .797 & 28,9 & 71.389 .377 .528 & 429.058 .800 & 170 \\
\hline Total (1) & 108.050 .528 & 2.580 .179 .790 & 12,7 & 389.133 .314 .142 & 2.338 .738 .320 & 151 \\
\hline
\end{tabular}

(1) N. ${ }^{\circ}$ DHDs, Media aritmética.

(2) Coste Tratamiento/Día en ptas. 
Figura 1

Consumo de Hipolipemiantes en España, 1997-2000, evolución por Subgrupos Terapéuticos ATC

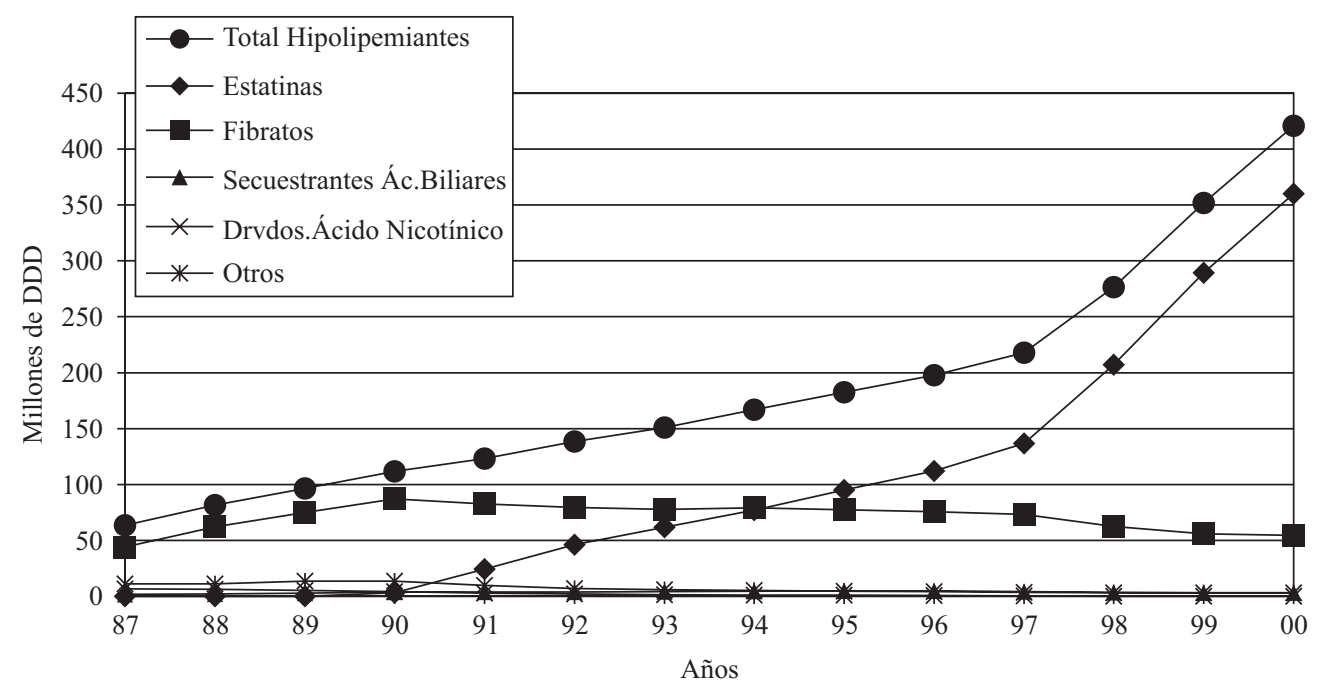

tanto estable, aunque con tendencia descendente que comienza justamente a partir de la aparición de las estatinas, si bien el incremento de estas últimas es muy superior al descenso de aquellos. En cuanto al resto de subgrupos, su presencia es casi inapreciable en comparación con los dos anteriores.

Para dimensionar la importancia relativa del consumo de hipolipemiantes en el Sistema Nacional de Salud, se calculó la proporción que suponen los hipolipemiantes sobre el total del consumo de especialidades farmacéuticas. Se usaron para ellos los parámetros $n .^{\circ}$ de envases e importe, ya que no nos fue posible calcular el consumo total de especialidades en $n .^{\circ}$ de DDD; o sea, el denominador de la relación. En n.$^{\circ}$ de envases, la evolución fue creciente de forma constante, yendo del 0,74 al 2,71\%. En cuanto al consumo en importe, la evolución se mantuvo siempre por encima de la proporción en envases y fue menos regular, con dos puntos de inflexión en 1991 y 1998; los valores anuales fueron los siguientes: 1,71, 2,07, 2,13, 2,32, 2,98, 3,36, 3,61, 3,83, 3,93, 3,95, $4,20,5,05,5,91$ у $6,25 \%$.
Se analizó la evolución de los principios activos del subgrupo con mayor consumo, el de las estatinas, representándose los resultados, expresados en n. ${ }^{\circ}$ de DDD, en la figura 2. Puede verse que la simvastatina fue la más consumida durante todo el período hasta 1999, cuando fue alcanzada por la atorvastatina, en el que era para ésta última el segundo año de vida en el mercado.

Para conocer los patrones de la situación actual del consumo, se halló el consumo de cada principio activo en el último año de estudio, el 2000. Los valores, expresados en n. ${ }^{\circ}$ de envases, $n .^{\circ}$ de DDD, n. ${ }^{\circ}$ de DHD e importe, así como el CTD se recogen en la tabla 3. También se indica la proporción que supone cada principio activo dentro del total de hipolipemiantes. Los principios activos están agrupados por subgrupos de la clasificación ATC, indicándose los subtotales de consumo de cada subgrupo. Puede verse que el subgrupo de mayor consumo es del las estatinas, y que dentro de éste destacan la atorvastatina y la simvastatina; con la circunstancia de que la segunda se encuentra en el mercado desde 1990 y la primera sólo 
Figura 2

Consumo de Hipolipemiantes en España, 1987-2000, Estatinas

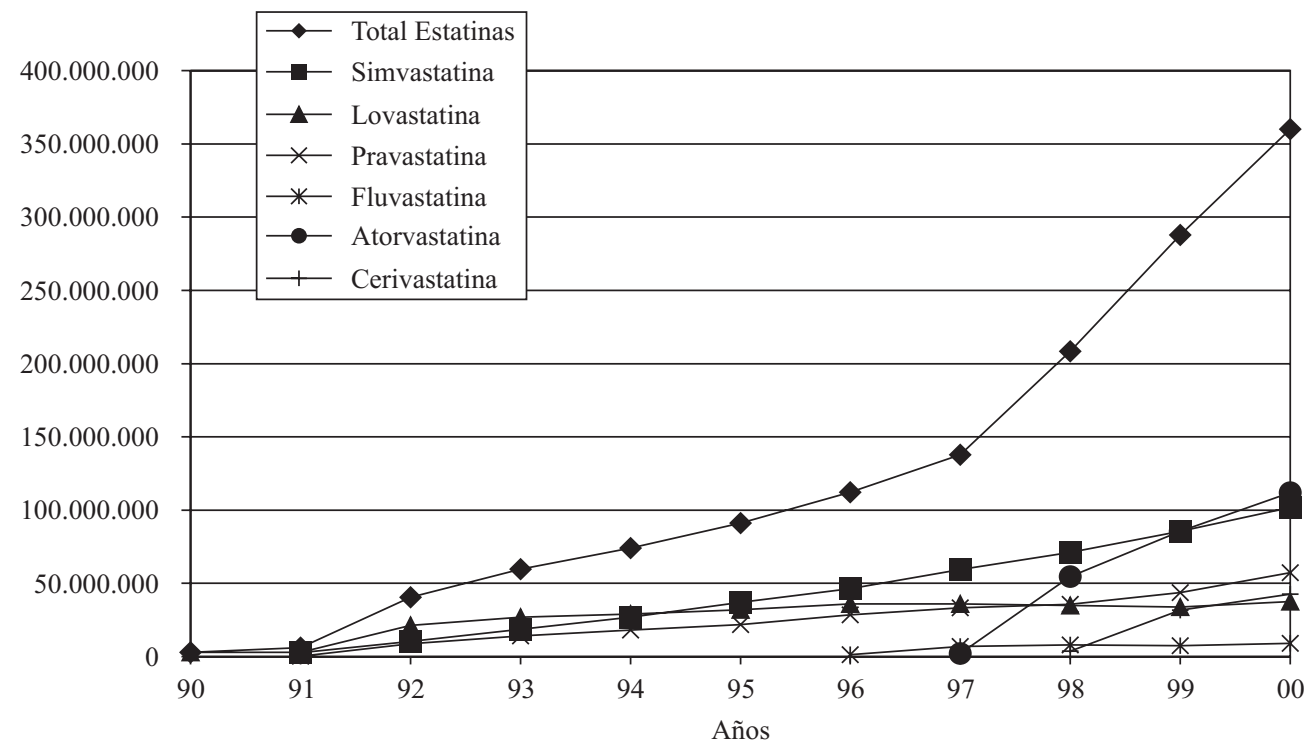

desde 1998. La diferencia es mayor en importe que en $n .^{\circ}$ de DDD debido al mayor CTD de la atorvastatina (182 ptas) respecto a la simvastatina (168 ptas). Entre ambos principios activos acumulan la mitad de todo el consumo de hipolipemiantes. Además, los cinco principios activos más consumidos son alguna de las seis estatinas que hay en el mercado: sólo la fluvastatina está por debajo de principios activos de otros subgrupos, siendo sobrepasada por tres fibratos: gemfibrozilo, fenofibrato y bezafibrato.

Teniendo en cuenta que el significado de la DDD es el de una media de consumo diario producido (y no una recomendación terapéu- tica), creemos que se pueden usar las DDD consumidas para hacer una estimación del n. ${ }^{\circ}$ de pacientes que están siendo tratados; especialmente en este caso en el que se tiene una amplia base, al tratarse de datos del total de España. Por ello, se calculó el n. ${ }^{\circ}$ estimado de pacientes en tratamiento con hipolipemiantes, dividiendo el $n .^{\circ}$ de DDD consumidas cada año por 365 días, y la proporción que representa sobre la población total española. Los resultados se recogen en la tabla 4; y en ella puede verse cómo se ha pasado de una proporción estimada de población en tratamiento del $0,45 \%$ en 1987 al $2,89 \%$ en el año 2000, con un n. ${ }^{\circ}$ estimado superior a 1.150.000 pacientes. En ese intervalo, la proporción no dejó de aumentar cada año. 
Tabla 3

Consumo de Hipolipemiantes en España en 2000, por principios activos

\begin{tabular}{|c|c|c|c|c|c|c|c|}
\hline & Envases & Importe & $N .^{o} D D D(1)$ & $\begin{array}{c}N .^{o} \\
D H D(2)\end{array}$ & $\begin{array}{l}\% / \text { total } \\
\operatorname{Imp}(3)\end{array}$ & $\begin{array}{c}\% / \text { total } \\
D D D(4)\end{array}$ & $C T D(5)$ \\
\hline Atorvastatina & 3.993 .179 & 20.332 .885 .595 & 111.809 .012 & 7,69 & 28,48 & 26,57 & 182 \\
\hline Simvastatina & 3.387 .947 & 17.070 .315 .223 & 101.825 .004 & 7,00 & 23,91 & 24,19 & 168 \\
\hline Pravastatina & 2.202 .239 & 12.130 .913 .571 & 57.357 .258 & 3,94 & 16,99 & 13,63 & 211 \\
\hline Cerivastatina & 1.545 .838 & 7.773.529.668 & 42.590 .296 & 2,93 & 10,89 & 10,12 & 183 \\
\hline Lovastatina & 1.875 .834 & 6.674 .787 .145 & 37.452 .856 & 2,57 & 9,35 & 8,90 & 178 \\
\hline Fluvastatina & 451.669 & 1.479 .585 .410 & 9.002 .154 & 0,62 & 2,07 & 2,14 & 164 \\
\hline Subtotal Estatinas & 13.456 .706 & 65.462 .016 .612 & 360.036 .580 & 24,75 & 91,70 & 85,54 & 182 \\
\hline Gemfibrozilo & 960.378 & 2.769 .777 .600 & 24.390 .891 & 1,68 & 3,88 & 5,80 & 114 \\
\hline Fenofibrato & 587.793 & 830.387 .692 & 20.719 .175 & 1,42 & 1,16 & 4,92 & 40 \\
\hline Bezafibrato & 438.337 & 762.338 .185 & 8.766 .740 & 0,60 & 1,07 & 2,08 & 87 \\
\hline Binifibrato & 24.647 & 69.130 .166 & 461.849 & 0,03 & 0,10 & 0,11 & 150 \\
\hline Clofibrato & 7.302 & 4.912 .228 & 154.644 & 0,01 & 0,01 & 0,04 & 32 \\
\hline Clofibrato de Aluminio en asociación & 1.844 & 1.416 .192 & 18.440 & 0,00 & 0,00 & 0,00 & 77 \\
\hline Tocofibrato & 1 & 1.125 & 25 & 0,00 & 0,00 & 0,00 & 45 \\
\hline Etofibrato & 1 & 994 & 20 & 0,00 & 0,00 & 0,00 & 50 \\
\hline Subtotal Fibratos & 2.020 .303 & 4.437.964.182 & 54.511 .784 & 3,75 & 6,22 & 12,95 & 81 \\
\hline Filicol & 61.154 & 109.159 .890 & 1.223 .080 & 0,08 & 0,15 & 0,29 & 89 \\
\hline Colestipol & 110.487 & 339.305 .577 & 828.653 & 0,06 & 0,48 & 0,20 & 409 \\
\hline Colestiramina & 45.689 & 85.261 .774 & 652.667 & 0,04 & 0,12 & 0,16 & 131 \\
\hline Dextrano Dietilaminoetil & 31.878 & 56.105 .280 & 318.780 & 0,02 & 0,08 & 0,08 & 176 \\
\hline Subtotal Secuestrantes Ác. Biliares & 249.208 & 589.832 .521 & 3.023 .180 & 0,21 & $\mathbf{0 , 8 3}$ & 0,72 & 195 \\
\hline Nicotinato de Tocoferol & 1.229 & 657.526 & 16.387 & 0,00 & 0,00 & 0,00 & 40 \\
\hline Pirozadilo & 16 & 44.443 & 450 & 0,00 & 0,00 & 0,00 & 99 \\
\hline $\begin{array}{l}\text { Nicotínico } \\
\text { Ácido + Pentosanopolisulfúrico }\end{array}$ & 8 & 6.096 & 160 & 0,00 & 0,00 & 0,00 & 38 \\
\hline Subtotal Drvdos. Ác. Nicotínico & 1.253 & 708.065 & 16.997 & $\mathbf{0 , 0 0}$ & 0,00 & $\mathbf{0 , 0 0}$ & 42 \\
\hline Sulodexida & 296.372 & 875.725 .727 & 2.929 .442 & 0,20 & 1,23 & 0,70 & 299 \\
\hline Pantetina & 5.101 & 16.796 .491 & 217.626 & 0,01 & 0,02 & 0,05 & 77 \\
\hline Benfluorex & 5.651 & 4.650 .773 & 113.020 & 0,01 & 0,01 & 0,03 & 41 \\
\hline Probucol & 878 & 728.956 & 13.200 & 0,00 & 0,00 & 0,00 & 55 \\
\hline Sultosilato de Piperazina & 563 & 571.445 & 8.445 & 0,00 & 0,00 & 0,00 & 68 \\
\hline Heparinoide & 779 & 359.440 & 8.275 & 0,00 & 0,00 & 0,00 & 43 \\
\hline Condroitinsulfúrico Ácido & 21 & 23.316 & 248 & 0,00 & 0,00 & 0,00 & 94 \\
\hline Subtotal Otros Hipolipemiantes & 309.365 & 898.856 .148 & 3.290 .256 & 0,23 & 1,26 & 0,78 & 273 \\
\hline Total Hipolipemiantes & 16.036 .835 & 71.389.377.528 & 420.878 .797 & 28,93 & 100,00 & 100,00 & 170 \\
\hline
\end{tabular}

(1) N. ${ }^{\circ}$ de Dosis Diarias Definidas.

(2) N. ${ }^{\circ}$ de Dosis Diarias Definidas / 1000 Habitantes / Día.

(3) Proporción sobre total hipolipemiantes en importe.

(4) Proporción sobre total hipolipemiantes en $n^{\circ}$ de DDD.

(5) Coste Tratamiento / Día. 
Tabla 4

Estimación del $n .^{\circ}$ de pacientes en tratamiento

\begin{tabular}{|c|c|c|c|}
\hline Año & $N .^{o} D D D s$ & $N .^{o}$ pacientes tratados & $\begin{array}{c}\text { Proporción / } \\
\text { población total }\end{array}$ \\
\hline 1987 & 63.594 .576 & 174.232 & $0,45 \%$ \\
\hline 1988 & 81.588 .267 & 223.529 & $0,58 \%$ \\
\hline 1989 & 96.440 .447 & 264.220 & $0,67 \%$ \\
\hline 1990 & 111.704 .547 & 306.040 & $0,77 \%$ \\
\hline 1991 & 123.005 .500 & 337.001 & $0,84 \%$ \\
\hline 1992 & 138.436 .252 & 379.277 & $0,98 \%$ \\
\hline 1993 & 150.935 .486 & 413.522 & $1,06 \%$ \\
\hline 1994 & 166.977 .420 & 457.472 & $1,15 \%$ \\
\hline 1995 & 182.633 .951 & 500.367 & $1,24 \%$ \\
\hline 1996 & 197.717 .830 & 541.693 & $1,34 \%$ \\
\hline 1997 & 217.842 .839 & 596.830 & $1,50 \%$ \\
\hline 1998 & 276.636 .159 & 757.907 & $1,91 \%$ \\
\hline 1999 & 351.787 .719 & 963.802 & $2,42 \%$ \\
\hline 2000 & 420.878 .797 & 1.153 .093 & $2,89 \%$ \\
\hline
\end{tabular}

\section{DISCUSIÓN}

En cuanto a los métodos de estudio, parece claro que la medición del consumo de medicamentos en $n .^{\circ}$ de DDD presenta ventajas sobre otros parámetros, razón por la que es más usado cada día ${ }^{24-27}$. El importe da sólo una idea de la significación económica del consumo y está sujeto a cambios puntuales y evoluciones de precios, lo que tiene una particular importancia cuando se analizan largos períodos de tiempo como en nuestro caso. El número de envases no mide con exactitud la cantidad de medicamento consumido, por cuanto en el mercado pueden y suelen existir varias presentaciones comerciales de un mismo medicamento con diferentes dosificaciones y/o número de unidades posológicas que, en sentido estricto, no se podrían sumar. Además, una evolución en el tiempo hacia el uso de envases más grandes o más pequeños podría dar la falsa impresión de estar produciéndose un menor o un mayor consumo.

Con los datos obtenidos, resulta evidente que los hipolipemiantes constituyen un grupo de medicamentos de gran importancia y de importancia creciente dentro de la tera- péutica actual. Ello se deduce tanto del constante aumento de su consumo en el período estudiado, en todas las unidades de medida que hemos usado, como en el incremento del porcentaje de consumo respecto del consumo total de especialidades farmacéuticas. Esta proporción resulta mayor si en vez de considerar la del período global se toma la actual, ya que las estatinas no han existido en el mercado más que a partir de 1990. También resulta evidente que ha aumentado el número de pacientes en tratamiento.

Las características principales de la evolución durante el período considerado quedan reflejadas en la tabla 2. Por una parte se puede constatar que existe un crecimiento constante en número de DHD hasta la fuerte inflexión al alza de 1998; a partir de aquí, en sólo tres años, de 1998 a 2000, prácticamente se triplica la cantidad que había en 1997. Por el contrario, en importe PVP se aprecia otra pequeña inflexión en 1991 y 1992, que se explica mediante el importante incremento del CTD de esos años. Esta evolución obedece a la introducción de las primeras estatinas en nuestro país en 1990 (lovastatina en julio y simvastatina en noviembre), de 
precio medio superior a los principios activos usados hasta entonces. Estas primeras estatinas han ido desplazando a todos los demás subgrupos de hipolipemiantes, como puede verse en el figura 1, habiendo casi desaparecido todos ellos a excepción de los fibratos. Al mismo tiempo, todas las estatinas han tenido una evolución creciente a lo largo de sus años de vida en el mercado, con la única excepción de la fluvastatina. Esto quiere decir que las nuevas moléculas no reemplazan a otras, sino que se suman. Aún así, puede hablarse de un incremento estable del consumo global en DHD, que no se alteró con la aparición de las estatinas, cuando el crecimiento era marcado por los fibratos. Pero a partir de 1997, año en que se introduce la atorvastatina (en octubre), el crecimiento se dispara tanto en $n .^{\circ}$ de DHD como en importe, sin duda debido al espectacular crecimiento que experimenta este principio activo (figura 2), que no fue parejo de una disminución de los otros principios activos preexistentes, que por el contrario siguieron creciendo al mismo ritmo, con la salvedad de la ralentización de la lovastatina y la fluvastatina. Por tanto, el consumo de la atorvastatina se suma de manera neta al consumo de los demás, que crece de modo constante año a año. También la cerivastatina, introducida en mayo de 1998 experimenta un despegue espectacular, aunque luego parece atenuarse un tanto. En resumen, la introducción de las primeras estatinas no alteró el crecimiento del número de tratamientos pero sí su coste, mientras que la atorvastatina y la cerivastatina dispararon tanto los tratamientos como el coste. Será interesante ver lo que ocurre en los próximos años con esta evolución.

El CTD muestra un estancamiento hacia el final del período de estudio, e incluso una disminución en el 2000 sobre 1999; esto podría deberse a medidas de tipo estructural adoptadas por el Ministerio de Sanidad y Consumo, como reducción de precios por disminución de márgenes comerciales del sector, aparición de especialidades farma- céuticas genéricas y aplicación del sistema de precios de referencia.

En cuanto a la estimación del número de pacientes que podrían estar tomando algún hipolipemiante calculado a partir de las DDD consumidas, a pesar de lo discutible que pueda parecer, creemos que, al tratarse de datos del total nacional, esos resultados deben estar muy promediados y que resulta lícito este planteamiento, con las lógicas reservas. Debe además tenerse en cuenta que estos tratamientos son siempre crónicos, lo cual ayuda a hacer válida esta aproximación. En cuanto a los resultados en sí (tabla 4), se aprecia claramente la creciente importancia de estos medicamentos, no sólo por las connotaciones económicas, si no por el número de personas a los que afectan. Esta tendencia creciente tiene, además, un notable punto de inflexión hacia arriba en 1998, lo que indicaría, de acuerdo también a otros datos ya comentados, que la aparición de la atorvastatina ha hecho tributario de tratamiento a un mayor número de personas, produciendo un espectacular cambio en este sentido, de modo que en sólo tres años (1998-2000), el número estimado de pacientes se ha duplicado.

Una de las indudables ventajas de usar la metodología ATC/DDD es la de poder hacer comparaciones internacionales de consumo, mediante el número de DHD. Este ejercicio sin embargo está plagado de problemas y limitaciones, no tanto por la metodología en sí, sino por la dificultad de equiparar los datos de consumo de los que se parte, que suele ser inherente al sistema público de salud del país de que se trate. En España tenemos un sistema bastante exacto derivado del proceso de facturación de las recetas médicas; esto nos proporciona datos de dispensación, que es lo más cercano a la administración al paciente; pero no nos permite tener en cuenta los medicamentos consumidos dentro del hospital, ni los usados por ciertos Regímenes Especiales de la Seguridad Social (minoritarios, es cierto) como MUFACE, ISFAS o MUGEJU; ob- 
viamente tampoco se consideran los envases consumidos sin receta o con receta privada. No obstante, en el grupo de medicamentos que nos ocupa, en nuestro país podemos suponer que el sesgo que se produce al usar datos de facturación de recetas de la Seguridad Social no es muy relevante, por cuanto se trata de medicamentos financiados, de uso ambulatorio y crónico, de receta médica obligatoria y de elevado precio, lo que creemos que disuadirá a muchos pacientes de obtenerlo sin financiación pública, y por tanto sin receta de la Seguridad Social. En otros países se toman datos de ventas de los laboratorios o de los distribuidores: en ambos casos se trata de eso, de ventas, y no de prescripciones o dispensaciones, conceptos éstos más cercanos al paciente y por tanto más ajustados al consumo real.

En cualquier caso, aunque las comparaciones entre países haya que hacerlas con cierta cautela, nos parece lícito establecerlas. Así, al confrontar los datos que estamos viendo para nuestro país con los de Noruega, publicados cada año por el Norsk Medisinaldepot $^{24}$, se aprecia también uno de estos puntos de inflexión, pero en el año 1995, debido a la simvastatina, aparecida en aquel país el año anterior. La evolución en este país es cuando menos sorprendente. Tradicionalmente, el consumo de estos medicamentos ha sido inferior en los países nórdicos que en otras zonas, incluida la mediterránea; $y$, ciertamente, hasta 1994 se mantuvo por debajo de las 5 DHD, cuando en España en ese año fue de 12,4 DHD. Pero a partir de ese año, en sólo tres más, en 1997 ha pasado a ser de 27,13, o sea, se ha multiplicado casi por 6; todo ello debido a la simvastatina. Estos crecimientos espectaculares han sido comunicados en otros estudios, por ejemplo, en Australia, donde se pasó de 1,2 DHD en 1987 a 5,2 DHD en $1989^{28}$.

En Estados Unidos, si bien se trata de datos no referidos a población, un estudio que hemos analizado ${ }^{29}$ recoge un incremento espectacular del consumo global de hipolipemiantes al final de la década de los 80 preci- samente por la introducción en aquel mercado de la primera estatina, la lovastatina. Resulta evidente que hoy en día, las estatinas han copado los tratamientos hipolipemiantes $^{30}$, y que su aparición en el arsenal terapéutico ha producido un mayor uso de este tipo de medicación, tanto en n. ${ }^{\circ}$ de DHD como en importe. Sólo los fibratos siguen teniendo un cierto papel.

Otro estudio comparativo de consumo en Australia, Italia, Suecia, Noruega y Finlandia $^{31}$ con datos de 1994 muestra un mayor consumo en Australia (11,9 DHD), seguida de Italia $(6,7)$, Suecia $(5,6)$, Noruega $(4,9)$ y Finlandia $(4,0)$; recordemos que el consumo en España fue para ese mismo año de 11,5 DHD. En el caso de Italia, no obstante, esta cifra estaría condicionada por un cambio en el esquema de financiación pública; habiendo descendido desde las 11,5 DHD de 1992 , cifra más equiparable a la de nuestro país, que en ese año fue de 9,8 DHD.

Igualmente, en otro estudio sobre las tendencias de consumo en los países nórdi$\cos ^{32}$, para el año 1993 se recogen valores desde 2,3 DHD hasta 4,0 DHD; en España, el consumo global para ese año fue bastante superior (10,6 DHD).

En Estados Unidos ${ }^{33}$, sin olvidar la dificultad de la comparación por las diferencias en la toma de datos, encontramos que la proporción que supusieron los hipolipemiantes sobre el total de ventas en 1992 fue del 3,7\%, similar al 3,36\% de España (medido en dispensaciones, no en ventas).

Otro estudio de consumo en nuestro país referido al período $1983-1991^{34}$ arroja datos similares a los de nuestro estudio, al tiempo que ponen de manifiesto el predominio de los fibratos antes de la introducción de las estatinas, y la tendencia a abandonar las asociaciones.

Sería muy interesante medir qué impacto ha tenido el uso de estos medicamentos sobre el curso de las enfermedades cardiovasculares, pero desgraciadamente ello está 
fuera de nuestro alcance. Esta severa limitación suele ser un factor común a la mayoría de los estudios de utilización de medicamentos, puesto que no existen registros estadísticos del diagnóstico y del curso de las patologías parejos a los del consumo de medicamentos.

\section{BIBLIOGRAFÍA}

1. Rodríguez Artalejo F, Rey Calero, J. La prevención secundaria de la enfermedad cardiovascular es prioritaria pero resulta insuficiente. Rev Esp Salud Publica, 1999;73:441-443.

2. Rodríguez Artalejo F, Guallar-Castillón P, Banegas Banegas JR, Rey Calero J. Trends in hospitalization and mortality for heart failure in Spain, 1980-1993. Eur Heart J 1997; 18:1771-1779.

3. Alonso Prieto I, Puchades Belenguer MJ. Evolución de la mortalidad por enfermedad cardiovascular en la provincia de Toledo, 1975-94. Rev Esp Salud Publica 1999;73:365-73.

4. Ministerio de Sanidad y Consumo, Sociedad Española de Cardiología y Sociedad Española de Arteriosclerosis. Control de la colesterolemia en España, 2000. Una herramienta para la prevención de la enfermedad cardiovascular. Rev Esp Salud Publica 2000;74:215-53.

5. Vega Alonso AT, Doménech Muñiz G, Melero Gonzalo M, Olmos Sanz A, Villar Cabeña A, Lozano Alonso E, Prieto Maricalva M. Riesgo de enfermedad isquémica del corazón en el área de salud de Valladolid Oeste. Rev Esp Cardiol 2000;53:353-359).

6. Balaguer-Vintro I, Sans S. Coronary heart disease mortality trends and related factors. Cardiology 1985;72(1-2):97-104.

7. Serra-Majem L, Ribas Treserras R, Ngo J, Salleras L. How could changes in diet explain changes in coronary heart disease mortality in Spain? The Spanish paradox. Am J Clin Nutr 1995;61 (6 Suppl):1351S-1359S.

8. Grupo de Trabajo de Prevención Cardiovascular. Guía de Prevención Cardiovascular. Programa de Actividades Preventivas y de Promoción de la Salud (PAPPS). Sociedad Española de Medicina Familiar y Comunitaria (SEMFYC) 1996.

9. Balaguer Vintro I. Estrategias en el control de los factores de riesgo coronario en prevención primaria y secundaria. Rev Esp Cardiol 1998;51 Suppl 6:30-5.
10. Kottke TE, Brekke MJ, Brekke LN, Dale LC, Brandel CL, DeBoer SW, Hayes SN, Hoffman RS, Menzel PA, Nguyen TT, Thomas RJ. The Cardiovision 2020 baseline community report card. Mayo Clin Proc 2000 Nov;75(11);1153-9.

11. Goodstein D, Faucard A, Peter D. Hipolipemiantes de elección. The Medical Letter. 1999; Vol XXI, N. ${ }^{\circ}$ 3:9-11.

12. Scandinavian Simvastatin Survival Study Group. Randomised trial of cholesterol lowering in 4444 patients with coronary heart disease: the Scandinavian Simvastatin Survival Study (4S). Lancet 1994;344:1383-1389.

13. Sacks et al. Cholesterol and Recurrent Event Trial Investigators (CARE). The effect of pravastatin on coronary events after myocardial infarction in patients with average cholesterol levels. $\mathrm{N}$ Engl J Med 1996; 335:1001-9.

14. Shepherd J, Cobbe SM et al. (The West of Scotland Coronary Prevention Study GroupWOSCOS). Prevention of coronary heart disease with pravastatin in men with hypercholesterolemia. N Engl J Med 1995;333:1301-1307.

15. Expert Panel on Detection, Evaluation and Treatment of High Blood Cholesterol in Adults. Summary of the second report of the National Cholesterol Education Program (NCEP). Expert Panel on Detection, Evaluation and Treatment of High Blood Cholesterol in Adults (Adult Treatment Panel II). JAMA 1993;269:3015-3023.

16. Prevention of coronary heart disease: scientific background and new clinical guidelines. Recommendations of the European Atherosclerosis Society prepared by the International Task Force for Prevention of Coronary Heart Disease. Nutr Metab Cardiovasc Dis 1992; 2:113-156.

17. Arnalich F. Tratamiento hipolipemiante: uso según factores de riesgo vascular. Ibf Ter Sist Nac Salud 1996; 20:1-7.

18. Sociedad Española de Medicina Familiar y Comunitaria. Dislipemias. Manejo de las dislipemias en Atención Primaria. Madrid: EMISA; 1997

19. Anatomical Chemical Classification Index with Defined Daily Doses (DDD's). Oslo: WHO Collaborating Centre for Drug Statistics Methodology, 2000 .

20. Reynolds JEF. Martindale. The Extra Pharmacopoeia. (31th ed.). London: The Pharmaceutical Press; 1996.

Rev Esp Salud Pública 2001, Vol. 75, N. ${ }^{\circ} 2$ 
21. Consejo General de Colegios Oficiales de Farmacéuticos. Catálogo de Especialidades Farmacéuticas 1998. Madrid: Consejo General de Colegios Oficiales de Farmacéuticos; 1998.

22. Medicom, S.A. Vademecum Internacional. Madrid: Ediciones Médicas, 1998.

23. García AG et al. Intercon 1996. Madrid: Editores Médicos, SA; 1997.

24. Norsk Medisinaldepot AS. Drug Consumption in Norway, 1993-1997. Oslo: NMD; 1998.

25. Goldaracena Tanco M, Hernández Dettoma, Obaldía Alaña C, Ortega Martínez J, Siles Gutiérrez M. Utilización de antiinfecciosos en la atención primaria de La Rioja. Aten Primaria 1998; 22:574-579.

26. Aza Pascual-Salcedo M. et al. Aplicación de la DDD en el estudio y consumo de antiinfecciosos. I Congreso de la Sociedad Española de Farmacéuticos de Atención Primaria. Jerez de la Frontera; 1996.

27. Siles Gutiérrez M et al. Impacto potencial de medidas de minimización de costes en la Prestación Farmacéutica en Atención Primaria de Aragón. Aten Primaria 1999; 23:352-358

28. Henry D, Cully LR, Grigson T, Lee C. Recent trends in prescribing of cholesterol lowering drugs in Australia. A report from the Drug Utilization Subcommittee of the Pharmaceutical Benefits Advisory Committee. Med J Aust 1991 Sep $2 ; 155(5) ; 332-6$.

29. Wysowski DK, Kennedy DL, Gross TP. Prescribed use of cholesterol lowering drugs in the United States, 1978 through 1988. JAMA 1990 Apr $25 ; 263(16): 2185-8$

30. Truter I, Kotze TJ. A drug utilization study investigating prescribed daily doses of hypolipidaemic agents. S Afr Med J 1996 Nov; 86(11):1397-401.

31. Magrini N, Einarson T, Vaccheri A, McManus P Montanaro N, Bergman U. Use of lipid-lowering drugs from 1990-1994: an international comparison among Australia, Finland, Italy (Emilia Romagna Region), Norway and Sweden. Eur J Clin Pharmacol 1997; 53(3-4):185-9.

32. Martikainen J, Klaukka T, Reunanen A, Peura S, Wahlroos H. Recent trends in the consumption of lipid-lowering drugs in Finland. J Clin Epidemiol 1996 Dec; 49(12):1453-57.

33. Use of cholesterol-lowering drugs, United States 1992. Stat Bull Metrop Insur Co Oct-Dec; 74(4):10-7.

34. De Abajo FJ, Madurga M, Montero D, Adin J, Palop R. Trends in the supply and use of lipid-lowering drugs in Spain, 1983-1991. Therapie 1993 Mar-Apr; 48(2):145-9. 